\title{
Alternative Directions to Control Spin Dynamics in Nuclear Magnetic Resonance and Physics
}

\author{
Eugene Stephane Mananga ${ }^{1,2,3 *}$
}

${ }^{1}$ Ph.D Program Physics \& Ph.D Program Chemistry, The Graduate Center, The City University of New York, USA

${ }^{2}$ Department of Applied Physics, New York University, USA

${ }^{3}$ Department of Engineering, Physics and Technology, The City University of New York, USA

As front-line theories to control spin dynamics in solid-state nuclear magnetic resonance, the Average Hamiltonian Theory (AHT) [1] and Floquet Theory (FLT) [2,3] have assumed great prominence and influence since the development of multiple pulse sequences and the inception of Magic-Angle Spinning (MAS) methods in the 1960s [4,5]. Methods developed over the past decade have enabled us to make a significant progress in the area of NMR by introducing an alternative expansion scheme called Floquet-Magnus Expansion (FME) [6,7] used to solve the time-dependent Schrodinger equation which is a central problem in quantum physics in general and solid-state NMR in particular. The FME establish the connection between the Magnus Expansion (ME) and the Floquet theory, and provides a new version of the ME well suited for the Floquet theory for linear ordinary differential equations with periodic coefficients [6-12]. We have proved that the ME is a particular case of the FME which yields new aspects not present in $\mathrm{ME}$ and Floquet theory such as recursive expansion scheme in Hilbert space that can facilitate the implementation of new or improvement of existing pulse sequences [6]. In the same vein, Madhu and Kurur has recently introduced the Fer Expansion (FE) in Solid-State NMR [13,14]. The Fer expansion was formulated by Fer [13] and later revised by Fer [13], Klarsfeld and Oteo [15], Casas, et al. [16] and Blanes, et al. [17]. This expansion employs the form of a product of sub-propagators, which appears to be suitable for examination of time-dependence of the density matrix for each average Hamiltonian at different orders. A paper which outlines the comparison of both theories (FME and FE) in
NMR and physics has recently been published in the Journal of Physical Chemistry A [18].

Using the FME and FE approaches, many problems can be attacked in other fields of physics beyond the scope of NMR. It is important to remember that these considered methods have recently found new major areas of applications such as topological materials [19].

However, researchers dealing with these new applications are not usually acquainted with the achievements of the magnetic resonance theory, where those methods were developed more than thirty years ago [10,20-22]. Researchers repeat the same mistakes that were made when the methods of spin dynamics and thermodynamics were developed in the past. Even though the FME is a divergent approach in general, its finite truncation can gives useful information such as on the transient dynamics in periodically driven many-body quantum systems [22,23]. New avenues of exploring FME and FE can also be extended to other areas of physics such as particles and high energy physics $[20,21]$. These two approaches (FME and FE) can be used to solve problems in Quantum Field Theory (QFT) and high energy physics, in particular problems similar to the one solved or fail to be solved by ME. For instance,

1. The ME has been used as an alternative to conventional perturbation theory for quantum fields to graph rules for functions of the time-evolution operator where normal products and Wick theorem were used. This was useful in the treatment of infrared divergences for some quan-

*Corresponding author: Eugene Stephane Mananga, Department of Engineering, Physics and Technology, The City University of New York, 2155 University Avenue, CPH 118, Bronx, 10453, New York, USA, Tel: +1-646-3454613, Fax: +1-718-289-6403, E-mail: esm041@mail.harvard.edu; emananga@gradcenter.cuny.edu

Received: July 06, 2017: Accepted: August 31, 2017: Published: September 02, 2017

Copyright: (c) 2017 Mananga ES. This is an open-access article distributed under the terms of the Creative Commons Attribution License, which permits unrestricted use, distribution, and reproduction in any medium, provided the original author and source are credited. 
tum electrodynamics process such as the scattering of an electron on an external potential or the bremsstrahlung of one hard photon [24,25]. I believe that effective method of approaching this problem demands more inspection where FME and FE can play a major role. The calculation of effective Hamiltonians has been used with great success in many areas of physics such as high resolution NMR spectroscopy [8-12], the topological states of Floquet in grapheme and semi-metals under the action of external electromagnetic fields [26-31], in superfluid systems [32-35], in systems discretized spatially by the presence of the lattice [36-38], in the periodically driven systems, among others. In the calculation of effective Hamiltonians, several different models are used, namely: the method of multi-temporal scales or the Floquet-Magnus and Fer expansions which are of our interest in this review. An infrared divergence in physics is a position in which an integral such as a Feynman diagram, diverges because of contributions of objects with very small energy approaching zero due to physical phenomena at very long distances. In particle physics, quantum electrodynamics is the relativistic quantum field theory of electrodynamics delineating how light and matter interact and is the first theory where full agreement between quantum mechanics and special relativity is concluded. The time independent Hamiltonian of FME [39-42] and FE [43] can then play a major role in the treatment of infrared divergences for some quantum electrodynamics process.

2. An extension of the ME has been applied to the context of Connes-Kreimer's Hopf algebra approach to perturbative renormalization of quantum field theory showing that the generalized ME [44,45] allows to solve the Bogoliubov-Atkinson recursion [46]. The FME and FE can also be applied in this context.

3. In the field of high energy physics, ME has also found applications such as to heavy ion collisions. ME is applied in collision problems when the use of unitary approximation scheme is necessary such as the unitary of the time evolution operator imposing some bound on the experimentally observable cross sections $[47,48]$. FME and FE can also be used in this context as an intuitive method for simplifying calculations.

4. The problem in neutron oscillations which is closely related to solar neutrino problem. As neutrinos with different masses propagate with different velocities, the mixing allows for flavor conversion corresponding to neutrinos oscillations [48-50]. Fer's factorization as a symplectic integrator can, in principle, enter in the solution of the evolution operator in one basis.

The introduction of FME and FE as theoretical approaches to control the spin dynamics in the field of nuclear magnetic resonance is a new exploratory and de- velopmental research which is a significant addition to the existing theoretical framework of AHT and FT. QFT is the basic mathematical language used to describe and analyze the physics of elementary particles. The theory by itself is an abstract representation for constructing quantum mechanics models of subatomic particles in particle physics and quasi particles in condensed matter physics. The application of the FME and FE approaches as intuitive approaches in simplifying calculations to solve some specifics problems in the field of high energy physics and QFT such as those outlined in the above paragraph is of major interest. It is worth noting that, the FME has the advantage of having the unitary character of the evolution operator which is preserved at all orders of approximation while the FE has an advantage over the ME that only an evaluation of nested commentators is required in the calculation of the Hamiltonian [14].

\section{References}

1. U Haeberlen, JS Waugh (1968) Coherent averaging effects in magnetic resonance. Phys Rev 175: 453-467.

2. MG Floquet (1883) Sur les équations différent ielleslinéaires à coefficients périodiques. Ann Econ Norm Suppl 12: 47-88.

3. JH Shirley (1965) Solution of the schrödinger equation with a hamiltonian periodic in time. Phys Rev B 138: 979-987.

4. ER Andrew, A Bradbury, RG Eades (1958) Nuclear magnetic resonance spectra from a crystal rotated at high speed. Nature 182: 1659.

5. J Lowe (1959) Free induction decays of rotating solids. Phys Rev Lett 2: 285-287.

6. ES Mananga, T Charpentier (2011) Introduction of the floquet-magnus expansion in solid-state nuclear magnetic resonance spectroscopy. J Chem Phys 135: 044109.

7. F Casas, JA Oteo, J Ros (2001) Floquet theory: exponential perturbative treatment. J Phys A: Math Gen 34: 3379-3388.

8. ES Mananga, AE Reid, T Charpentier (2012) Efficient theory of dipolar recoupling in solid-state nuclear magnetic resonance of rotating solids using Floquet-Magnus expansion: application on BABA and $\mathrm{C} 7$ radio frequency pulse sequences. Solid State Nucl Magn Res 41: 32-47.

9. S Blanes, F Casas, JA Oteo, J Ros (2009) The magnus expansion and some of its applications. Phys Rep 470: 151-238.

10. El Kuznetsova, EB Feldman, DE Feldman (2016) Magnus expansion paradoxes in the study of equilibrium magnetization and entanglement in multi-pulse spin locking. Phys Usp 59: 577-582.

11. Mananga ES, Hu B (2016) Controlling the dynamics of quadrupolar spin-1 nuclei by means of average hamiltonian theory when irradiated with modified composite quadrupolar echo pulse sequences. J Phys Chem A 120: 8657-8679.

12. Feldman EB (1984) On the convergence of the magnus expansion for spin systems in periodic magnetic fields. Phys Lett A 104: 479-481.

13. F Fer (1958) Resolution of the matrix equation $U=p U$ by product in final matrix expenses. Bull Class Sci Acad $R$ Belg 44: 818-829. 
14. PK Madhu, ND Kurur (2006) Fer expansion for effective propagators and Hamiltonians in NMR. Chem Phys Lett 418: 235-238.

15. S Klarsfeld, JA Oteo (1989) Exponential infinite-product representations of the time-displacement operator. J Phys A: Math Gen 22: 2687-2694.

16. F Casa, JA Oteo, J Ros (1991) Lie algebraic approach to Fer's expansion for classical Hamiltonian systems. J Phys A: Math Gen 24: 4037-4046.

17. S Blanes, F Casas, JA Oteo, J Ros (1998) Magnus and Fer expansions for matrix differential equations: the convergence problem. J Phys A: Math Gen 31: 259-268.

18. ES Mananga (2013) Applications of floquet-magnus expansion, average hamiltonian theory and fer expansion to study interactions in solid state $\mathrm{nmr}$ when irradiated with the magic-echo sequence. Solid State Nucl Magn Reson 55-56: 54-62.

19. T Mikama, S Kitamura, K Yasuda, N Tsuji, T Oka, et al. (2016) Brillouin-wigner theory for high-frequency expansion in periodically driven systems: Application to floquet topological insulators. Phys Rev B 93: 144307.

20. Es Mananga, T Charpentier (2016) On the floquet-magnus expansion: Applications in solid-state nuclear magnetic resonance and physics. Phys Rep 609: 1-49.

21. ES Mananga (2016) On the Fer expansion: Applications in solid-state nuclear magnetic resonance and physics. Phys Rep 608: 1-41.

22. Eckardt, E Anisimovas (2015) High-frequency approximation for periodically driven quantum systems from a Floquet-space perspective. New J Phys 17: 093039.

23. T Kuwahara, T Mori, K Saito (2016) Floquet-Magnus theory and generic transient dynamics in periodically driven many-body quantum systems. Annals of Physics 367: 96-124.

24. HD Dahmen, T Mannel, P Manakos (1988) Practical application of the unitary approximation to infrared singularities. Phys Rev D Part Fields 38: 1176-1182.

25. HD Dahmen, P Manakos, T Mannel, T Ohl (1991) Unitary approximation for radiative high-energy scattering processes: application to Bhabha scattering. Z Phys C 50: 75-84.

26. NH Lindher, Doron L Bergman, G Refael, V Galitski (2013) Topological floquet spectrum in three dimensions via a two-photon resonance. Phys Rev B 87: 235131.

27. YH Wang, H Steinberg, P Jarillo-Herrero, N Gedik (2013) Observation of floquet-bloch states on the surface of a topological insulator. Science 342: 453-457.

28. P Delplace, A Gomez-Leon, G Platero (2013) Merging of dirac points and floquet topological transitions in AC-driven grapheme. Phys Rev B 88: 245422.

29. G Grushin, A Gomez-Leon, T Neupert (2014) Floquet fractional chern insulators. Phys Rev Lett 112: 156801-156805.

30. Gomez Leon, P Delplace, G Platero (2014) Engineering anomalous quantum Hall plateaus and anti-chiral states with AC fields. Phys Rev B 89: 205408.

31. Titum $P$, Lindner NH, Rechtsman MC, Refael G (2015) Disorder-induced floquet topological insulators. Phys Rev Lett 114: 056801.

32. L Jiang, T Kitagawa, J Alicea, Ar Akhmerov, D Pekker, et al. (2011) majorana fermions in equilibrium and in driven cold-atom quantum wires. Phys Rev Lett 106: 220402.

33. G Liu, N Hao, SL Zhu, WM Liu (2012) Topological superfluid transition induced by a periodically driven optical lattice. Phys Rev A 86: 013639.

34. QJ Tong, JH An, J Gong, HG Luo, CH Oh (2013) Generating many majorana modes via periodic driving: A superconductor model. Phys Rev B 87: 201109.

35. M Thakurathi, AA Patel, D Sen, A Dutta (2013) Floquet generation of Majorana end modes and topological invariants. Phys Rev B 88: 155133.

36. LA Martınez, D Sanjines, JP Gallinar (2014) Semi classical tight-binding dynamics in rapidly oscillating fields plus a static arbitrary potential. Int J Mod Phys B 28: 1450173.

37. AP Itin, AI Neishtadt (2014) Effective hamiltonians for fastly driven tight-binding chains. Phys Lett A 378: 822-825.

38. Eckardt, E Anisimovas (2015) High-frequency approximation for periodically driven quantum systems from a Floquet-Space Perspective. New J Phys 17: 093039.

39. ES Mananga, T Charpentier (2015) Floquet-magnus expansion for general $n$-coupled spins systems in magic-angle spinning nuclear magnetic resonance. Chem Phys 450-451: 83-90.

40. ES Mananga (2013) Progress in spin dynamics solid-state nuclear magnetic resonance with the application of Floquet-Magnus expansion to chemical shift anisotropy. Solid State Nucl Magn Reson 54: 1-7.

41. ES Mananga (2013) Criteria to average out the chemical shift anisotropy in solid-state $\mathrm{nmr}$ when irradiated with BABA I, BABA II, and C7 radiofrequency pulse sequences. Solid State Nucl Magn Reson 55-56: 63-72.

42. ES Mananga, AE Reid (2013) Investigation of the effect of finite pulse errors on BABA pulse sequence using floquet-magnus expansion approach. Mol Phys 111: 243-257.

43. ES Mananga, J Moghaddasi, A Sana, A Akinmoladun, M Sadoqi (2015) Advances in theory of solid-state nuclear magnetic resonance. J Nat Sci 1: e109.

44. Connes A, D Kreimer (2000) Renormalization in quantum field theory and the riemann-hilbert problem i: the hopf algebra structure of graphs and the main theorem. Commun Math Phys 210: 249-273.

45. Connes A, D Kreimer (2001) Renormalization in quantum field theory and the Riemann-Hilbert problem II: the $\beta$-function, diffeomorphisms and the renormalization group. Commun Math Phys 216: 215-241.

46. K Ebrahimi Fard, D Manchon, F Patras (2008) New identities in dendriform algebras. J Algebra 320: 708-727.

47. DC lonescu (1994) Unitarity and electron-positron pairs created by strong external fields. Phys Rev A 49: 3188.

48. JC DOlivo (1992) Magnus approximation for nonadiabatic transitions and neutrino oscillations in matter. Phys Rev D 45: 924-930.

49. JC DOlivo, JA Oteo (1996) Nonadiabatic three neutrino oscillations in matter. Phys Rev D 54: 1187-1193.

50. AD Supanitsky, JC DOlivo, G Medina Tanco (2008) Perturbative exponential expansion and matter neutrino oscillations. Phys Rev D 78: 045024. 